\title{
Ankyrin2 is required for neuronal morphogenesis and long- term memory and interacts genetically with HDAC4
}

\author{
Silvia Schwartz ${ }^{\dagger}$, Sarah J Wilson ${ }^{\dagger}$, Tracy K Hale and Helen L Fitzsimons ${ }^{{ }^{*}}$ \\ ${ }^{\dagger}$ These authors contributed equally \\ ${ }^{1}$ Biochemistry, Biotechnology and Biomedical Science Group, School of Fundamental \\ Sciences, Massey University, Palmerston North, New Zealand \\ * Correspondence: \\ Helen Fitzsimons \\ h.l.fitzsimons@massey.ac.nz
}

\author{
Keywords: Ankyrin repeat, Ankyrin2, ANK3, histone deacetylase, HDAC4, memory, \\ neuron, Drosophila, mushroom body
}

\begin{abstract}
Dysregulation of HDAC4 expression and/or subcellular distribution results in impaired neuronal morphogenesis and long-term memory in Drosophila melanogaster. A recent genetic screen for genes that interact in the same molecular pathway as HDAC4 identified the cytoskeletal adapter Ankyrin2 (Ank2). Here we sought to investigate the role of Ank2 in neuronal morphogenesis, learning and memory, and to examine the nature of interaction with HDAC4. We found that Ank2 is expressed widely throughout the Drosophila brain where it localizes predominantly to axon tracts. Pan-neuronal knockdown of Ank2 in the mushroom body, a region critical for memory formation, resulted in defects in axon morphogenesis, and similarly reduction of $A n k 2$ in lobular plate tangential neurons of the optic lobe disrupted dendritic branching and arborization. Conditional knockdown of Ank2 in the mushroom body of adult Drosophila significantly impaired long-term courtship memory, and this requirement for Ank2 was isolated to gamma ( $\gamma$ ) neurons of the mushroom body. As overexpression of HDAC4 in $\gamma$ neurons also impairs the formation of long-term courtship memory, this suggests that any functional relationship between these proteins during LTM likely occurs in $y$ neurons. We determined that the genetic interaction requires the presence of nuclear HDAC4 and is not dependent on a conserved putative ankyrin-binding motif present in HDAC4. In summary, we provide the first characterization of the expression pattern of Ank2 in the adult Drosophila brain and demonstrate that Ank2 is critical for morphogenesis of the mushroom body and for the molecular processes required in the adult brain for formation of long-term memories.
\end{abstract}

\section{Introduction}

Histone deacetylase 4 (HDAC4) is a member of the Class lla family of histone deacetylases, which are categorized by the presence of an extended N-terminal regulatory region and their ability to undergo nucleocytoplasmic shuttling (GROZINGER AND SCHREIBER 2000; MCKINSEY et al. 2000; WANG et al. 2000; WANG AND YANG 2001; CHAWLA et al. 2003; SCHLUMM et al. 2013). HDAC4 is expressed widely throughout the brain and dysregulation of HDAC4 expression and/or 
subcellular distribution has been implicated in several neurodevelopmental and neurodegenerative disorders (WILLIAMS et al. 2010b; Li et al. 2012; SANDO et al. 2012; HeRruP et al. 2013; MielCAREK et al. 2013; SHEN et al. 2016; TRAZZI et al. 2016). Loss of HDAC4 is associated with Chromosome 2 q37 deletion syndrome [MIM 600430], the clinical features of which include developmental delay, autistic features and intellectual disability (WILLIAMS et al. 2010b; WILLIAMS et al. 2010a; MoRRIS et al. 2012a; MorRIS et al. 2012b; VILLAVICENCIO-LORINI et al. 2013). Developmental delay is the most common feature, and while haploinsufficiency of HDAC4 displays variable penetrance with respect to intellectual disability (WHEELER et al. 2014), the predominant genetic cause is considered to be loss of function of HDAC4 (DAG et al. 2019). HDAC4 has been shown to regulate synaptic plasticity and memory formation in several animal models (WANG et al. 2011; KIM et al. 2012a; SANDO et al. 2012; FITZSIMONS et al. 2013; SCHWARTZ et al. 2016; MAIN 2021); In mice, brain-specific conditional knockout of HDAC4 results in impaired spatial memory (KIM et al. 2012b; KIM et al. 2012a) and in Drosophila, RNAi knockdown of HDAC4 in the brain also impairs long-term memory (LTM) formation (FITZSIMONS et al. 2013). Disruption of normal subcellular distribution is also detrimental to neurodevelopment and cognition; a recent study identified seven individuals presenting with developmental delay, intellectual disability and other neurodevelopmental deficits, all of whom had de novo mutations in the 14-3-3 binding site within HDAC4. This motif is required for phosphorylation-dependent nucleocytoplasmic shuttling of HDAC4, and thus the disruption to 14-3-3 binding is presumed to disrupt normal HDAC4 shuttling, leading to nuclear accumulation (WAKELING et al. 2021). In mice, a truncated mutant of HDAC4 that accumulates in the nucleus causes deficits in spatial memory. This mutant lacks the deacetylase domain (SANDO et al. 2012), however vertebrate HDAC4 is catalytically inactive (LAHM et al. 2007; BOtTOMLEY et al. 2008), reviewed by (FITZSIMONS 2015). Similarly, overexpression of HDAC4 in the adult mushroom body, a brain region essential for memory formation in Drosophila (HEISENBERG et al. 1985; MCBRIDE et al. 1999), prevented LTM formation, as did a catalytically inactive mutant (FITZSIMONS et al. 2013). This memory phenotype was recapitulated with a mutant of HDAC4 that is restricted to the nucleus, and when expressed during development it also impaired morphogenesis of mushroom body axons (MAIN 2021).

Increased nuclear HDAC4 has also been observed in hippocampal pyramidal neurons in mouse models of Alzheimer's disease (SHEN et al. 2016). Moreover, HDAC4 was found to accumulate in pyramidal cells and layer III of the frontal cortex in postmortem brains from individuals with Alzheimer's disease, with the abundance of nuclear HDAC4 correlating with the clinical dementia scores (HERRUP et al. 2013; SHEN et al. 2016). These data together indicate that nuclear HDAC4 impairs cognitive function and that dysregulation of nucleocytoplasmic shuttling contributes to disease progression.

To gain further knowledge of the molecular pathway through which HDAC4 acts, we previously carried out a genetic enhancer screen in Drosophila photoreceptors for genes that interact genetically with HDAC4 (SCHWARTZ et al. 2016). Expression of HDAC4 in the eye results in a mild "rough" eye phenotype, as characterized by disruption of the regular ommatidial patterning. We identified a group of cytoskeletal 
regulators including Ankyrin2 (Ank2), that when knocked down in combination with HDAC4 overexpression resulted in an enhanced rough eye phenotype, indicative of a genetic interaction. We focused our attention on further investigating Ank2, since the altered expression of the human homologue $A N K 3$ has been associated with a variety of neurodevelopmental disorders including intellectual disability, epilepsy, attention deficit hyperactivity disorder (IQBAL et al. 2013), bipolar disorder (TESLI et al. 2011) and autism spectrum disorder ( $\mathrm{Bl}$ et al. 2012). Moreover, ANK3 single nucleotide polymorphisms have been associated with schizophrenia (ATHANASIU et al. 2010; YUAN et al. 2012; NiE et al. 2015a; Guo et al. 2016; HUGHES et al. 2018) and Alzheimer's disease (MORGAN et al. 2008).

Ankyrins are adapter proteins that link the underlying spectrin-actin cytoskeleton to integral membrane proteins such as ion channels, anion exchangers, signaling proteins and cell adhesion molecules (BENNETT 1978; BENNETt AND BAINES 2001; Mohler et al. 2002). Canonical ankyrins are comprised of an $\mathrm{N}$-terminal membrane binding domain, which contains 24 ANK repeat motifs organized as two anti-parallel $\alpha$-helices that mediate protein-protein interactions. They also contain a central spectrin-binding domain, a death domain and a less conserved C-terminal regulatory domain (SEDGWICK AND SMERDON 1999; CUNHA AND Mohler 2009). Drosophila Ank2 displays high similarity to human ANK3 (IQBAL et al. 2013), sharing 57\% amino acid identity over the whole protein and $71.2 \%$ identity across the ankyrin repeat region. Similarly to $A N K 3$, expression of $A n k 2$ is restricted to neurons and a number of transcript isoforms are also expressed. In the embryonic nervous system, shorter isoforms of Ank2 (Ank2-S) localize to the cell bodies of neurons, whereas medium Ank2-M isoforms localize to axons and are essential for viability (HORTSCH et al. 2002). A longer Ank2-L isoform containing an additional C-terminal domain localizes to axons and synaptic boutons at the neuromuscular junction. Mutants with disrupted Ank2-L expression display a loss of synapse stability as well as a reduction in the terminal bouton size, disassembly of presynaptic active zone and retraction of the synaptic microtubule cytoskeleton ( $\mathrm{KOCH}$ et al. 2008; PIELAGE et al. 2008), thus Ank2-L is required for synapse stability and normal morphology at the neuromuscular junction in Drosophila larvae.

Despite comprehensive investigation of the roles that Ank2 plays in neuronal development, the role of Ank2 in the adult brain and functional consequences of reduced Ank2 has received little attention. Here we aimed to investigate the role of Ank2 in learning, memory and development of the Drosophila brain as well as to examine the nature of the interaction between HDAC4 and Ank2 and to determine whether this is an important mechanism through which HDAC4 regulates neuronal development and memory.

\section{METHODS}

\section{Fly strains}

All flies were raised on standard medium on a 12-hour light/dark cycle and maintained at a temperature of $25^{\circ} \mathrm{C}$ unless otherwise indicated. $w\left[{ }^{*}\right]$; $P\{w[+m W . h s]=G a w B\} O K 107$ ey[OK107]/In(4)ci[D], ci[D] pan[ciD] sv[spa-pol] (OK107GAL4), $\left.\quad w{ }^{*}\right] ; \quad P\{w[+m C]=G A L 4-n i n a E . G M R\} 12 \quad$ (GMR-GAL4), $P\{w[+m W . h s]=G a w B\}$ elav[c155] (elav-GAL4), P\{w+mW.hs=GawB\}c739 (c739-GAL4), 
$w^{1118} ; P\{w+m W . h s=G a w B\} c 305 a(c 305 a-G A L 4), w^{1118} ; P\{w+m W . h s=G a w B\} 1471(1471-$ GAL4), $\mathrm{w}$ [1118]; P\{y[+t7.7] $\mathrm{w}[+\mathrm{mC}]=\mathrm{GMR} 16 A 06-G A L 4\} a t t P 2 \quad(R 16 A 06-G A L 4)$ and $P\{w[+m W . h s]=G a w B\} 3 A(3 A-G A L 4)$ were obtained from the Bloomington Drosophila Stock Center. $\left.w{ }^{*}\right] ; P\{w\{+m W . h s]=G a w B\} N P 1131$ (NP1131-GAL4) and $w[1118]$; PBac\{EGFP-IV\}ank2[KM0104] (Ank2::EGFP) were obtained from the Kyoto Stock Center. $P\{w+m C=$ tubP-GAL80ts $\} 10$ (tubP-GAL80 ${ }^{\text {ts }}$ ), p\{MEF2-GAL4.247\}(MB247-GAL4) and $w(C S 10)$ strains were kindly provided by R. Davis (The Scripps Research Institute, Jupiter, FL). P\{KK106729\}VIE-260B (UAS-Ank2 RNAi, VDRC ID 107369) and $w^{1118} ; P\{G D 12247) v 40638$ (UAS-Ank2 RNAi, VDRC ID 40638) were obtained from the Vienna Drosophila Resource Center. All strains were outcrossed for a minimum of five generations to $w(C S 10)$ flies. A homozygous line harbouring $w(C S 10) ; P\{w+m C=t u b P$ GAL80ts $\} 10$ and $P\{w+m W . h s=G a w B\} O K 107$ (tubP-GAL80ts; OK107-GAL4) was generated by standard genetic crosses, as was (elav-GAL4; tubP-GAL80ts), (c739-GAL4; tubP-GAL80 ts ), (tubP-GAL80 ${ }_{t s,}$ 1471-GAL4), (tubP-GAL80ts; MB247-GAL4), (tubPGAL80 $t s$, c305a-GAL4) (tubP-GAL80ts; R16A06-GAL4) and (tubP-GAL80ts, NP1131GAL4). UAS-DmHDAC4 and UAS-DmHDAC4 3SA have been described previously (MAIN 2021). UAS-DmHDAC4 $\triangle A n k$ was generated by site directed mutagenesis of UASDmHDAC4 with the following amino acid substitutions: P48A L50A P51A and I53A. The UAS-Ank2 ${ }_{190-946}-H A$ construct consists of a $2268 \mathrm{bp} \mathrm{N}$-terminal region of Ank2 containing the ankyrin repeat region (nucleotides 1123 - 3393 of Ank2, NCBI reference NM_001274607, which corresponds to amino acids 190 - 946). This construct with a C-terminal 3x HA epitope tag was generated and subcloned into pUASTattB by Genscript (NJ, USA). Transgenic flies were generated by Genetivision (Houston, TX, USA) using the VK22 docking site at 2R(57F5).

\section{Immunohistochemistry}

Whole flies were fixed in PFAT/DMSO (4\% paraformaldehyde in $1 \mathrm{X}$ PBS $+0.1 \%$ Triton $X-100+5 \%$ DMSO) for one hour then brains were microdissected in $1 \mathrm{X}$ PBS. Brains were post-fixed in PFAT/DMSO for 20 mins and blocked in immunobuffer (5\% normal goat serum in $1 \mathrm{X}$ PBS $+0.5 \%$ Triton $\mathrm{X}-100)$ for three hours prior to incubation with primary antibody of rabbit anti-Ank2-L (1:1000, gift from $\mathrm{H}$. Aberle) (КоCH et al. 2008), rabbit anti-GFP (Abcam, ab290 1:10,000), mouse anti-nc82 (1:100), mouse anti-Futsch (1:20), mouse anti-Repo (1:20) and mouse anti-Fasciclin II (Fas II, 1:200). Brains were then incubated with secondary antibody (goat anti-mouse Alexa 488 or 555, or goat anti-rabbit Alexa 488 or 555, Sigma Aldrich, 1:500) and mounted with Antifade. The monoclonal antibodies anti-Brp (nc82, developed by E. Buchner), anti-futsch (22C10, developed by S. Benzer and N. Colley), anti-Repo (8D12, developed by C. Goodman), and anti-Fasll (1D4, developed by C. Goodman) were obtained from the Developmental Studies Hybridoma Bank developed under the auspices of the NICHD and maintained by The University of lowa, Department of Biology, lowa City, IA 52242. For confocal microscopy, images were captured with a Leica TCS SP5 DM6000B Confocal Microscope and images were processed with Leica Application Suite Advanced Fluorescence (LAS AF) software and Image $J(N I H)$. For quantification of 180 dendrite branch length, the total shaft and major branch length inclusive of all six 181 neuronal shafts and their major visible branches were traced using the SNT program 182 in the ImageJ NeuroAnatomy plugin, which allows branching trace plots to be 183 reproduced from the dendritic arborizations (Avery et al., 2017). Total shaft and 
branch lengths were traced and these measurements were then added together to produce a total sum branch length. Statistical analysis was carried out with the student's $t$-test with significance set at $p<0.05$.

\section{Western Blotting}

Whole cell extracts were prepared from 100 snap-frozen heads by homogenizing in RIPA buffer, followed by centrifugation at $13,000 \mathrm{~g}$ for 2 minutes at $4^{\circ} \mathrm{C}$. Lysates ( 30 $\mu \mathrm{g})$ were resolved on 4-20\% SDS-PAGE gels (Biorad) and transferred onto nitrocellulose membranes. Membranes were blocked for $>1$ hour at room temperature in $5 \%$ skim milk powder in TBST $(50 \mathrm{mM}$ Tris, $150 \mathrm{mM} \mathrm{NaCl}, 0.05 \%$ Tween-20, pH 7.6) then incubated overnight at $4^{\circ} \mathrm{C}$ in primary antibody, washed $3 \times 5$ mins in TBST then incubated one hour in secondary anti-mouse, anti-rat or anti-rabbit HRP-conjugated antibodies (GE Life Sciences) as appropriate. Following $3 \times 5 \mathrm{~min}$ washes in TBST, proteins were detected with Amersham ECL Prime (GE Life Sciences). The following antibodies were used: rabbit anti-GFP (Abcam ab290, 1:10,000); rabbit anti-Myc (Roche, 1:1,000); rat anti-HA (Roche, 1:1,000) and mouse anti- $\alpha$-tubulin (12G10 clone, DSHB, 1:500).

\section{Co-immunoprecipitation}

Whole cell extracts were prepared as per the western blotting method above. Immunoprecipitation (IP) was performed with the Pierce Classic IP Kit (Thermo Scientific) according to the manufacturer's instructions. Anti-Myc or anti-HA antibody $(1 \mu \mathrm{L})$ was incubated overnight with $1 \mathrm{mg}$ of lysate. Following elution in $2 \mathrm{x}$ sample buffer, IP samples were processed for SDS-PAGE and western blotting with anti-HA or anti-Myc alongside $30 \mu \mathrm{g}$ input samples. Anti- $\alpha$-tubulin (1:500) was used as a loading control.

\section{RT-qPCR}

elav-GAL4 females were crossed to UAS-Ank2 RNAi males to generate progeny in which Ank2 was knocked down in all neurons; and progeny of elav-GAL4 crossed to $w(C S 10)$ served as the control. To confirm knockdown, total RNA was extracted from Drosophila heads from three independent crosses with the RNeasy Mini kit (Qiagen) according to the manufacturer's instructions. CDNA was synthesized from $1 \mu \mathrm{g}$ of total RNA with Transcriptor (Roche) as per the manufacturer's instructions. RT-qPCR was conducted using SsoFast-EvaGreen (BioRad) reaction master on a Lightcycler II 480 instrument (Roche), following manufacturer's instructions. The following primers were used: Ank2for 5'-GGCCGATATGGCACAAAACC-3', Ank2rev 5'TTCTTTCGACGGTGGTACGG-3', EF1a48Dfor 5'-ACTTTGTTCGAATCCGTCGC-3', EF1a48Drev 5'-TACGCTTGTCGATACCACCG-3'. A 5-fold dilution of cDNA from control flies was used as template to prepare a standard curve to confirm efficiency of the PCR reactions. Relative quantification was conducted using $2^{-\Delta \Delta C t}$ method, normalizing to the housekeeping gene Ef1 $148 D$ (LIVAK AND SCHMITTGEN 2001). Ank2 expression was reduced to $0.42 \pm 0.12$ (mean \pm standard error) of that of the control, student's $t$-test $t_{(12)}=4.74, p<0.001$.

\section{Courtship Suppression Assay}


230 The repeat training courtship suppression assay was used to assess 24-hour long-term

231 courtship memory. This is an experience-dependent assay in which wild-type male 232 flies that have been previously rejected by a mated female will reduce their courtship 233 behavior towards a new mated female. During mating, the male pheromone cVA is 234 transferred to the female, and the presence of this pheromone on the female causes 235 the male to reduce his courtship towards her. Males that have previously experienced 236 rejection will suppress courtship towards another mated female due to an enhanced 237 response to cVA (EJIMA et al. 2005; KELEMAN et al. 2007) and this form of courtship 238 memory is termed cVA-retrievable memory (RAUn et al. 2021). The detailed 239 methodology has been described previously (FITZSIMONS AND SCOTT 2011; FITZSIMONS et 240 al. 2013; FREYMUTH AND FITZSIMONS 2017). For training, single virgin males (3-5 days post 241 eclosion) of each genotype were placed into individual training chambers. A freshly 242 mated wild-type female was placed with each male to be trained, whereas sham 243 control males were housed alone. Over the seven-hour training period, multiple bouts 244 of courtship were observed in the trained group. The female fly was then aspirated 245 from the training chamber and the males were left in their chambers for the 24 hours, prior to testing. Each trained or sham male fly was then placed into a testing chamber containing a mated wild-type female and was scored for the time spent performing stereotypic courtship behaviors over the ten-minute period. A courtship index $(\mathrm{Cl})$ was calculated as the proportion of the ten-minute period spent courting. A mean $\mathrm{Cl}$ for each group was determined, and from this a memory index (MI) was calculated by the following equation: $\mathrm{MI}=1-(\mathrm{Cl}$ of each trained fly/mean $\mathrm{Cl}$ of sham group) ( $n \geq 16 /$ group). The Ml was measured on a scale of 0 to 1 , a score of 0 indicating memory was no different than untrained sham controls. In all experiments, the scorer was blind to the genotype of the flies. For assessment of immediate short-term memory, the training session was reduced to one hour and flies were tested immediately after training. For assessment of learning, the male was placed with a mated female for an hour and the first ten minutes and last ten minutes were scored for courtship behavior. The learning index was calculated as $1-(\mathrm{Cl}$ last $10 \mathrm{mins} / \mathrm{Cl}$ first 10 mins). For statistical analyses, data was arcsine transformed to approximate a normal distribution and one-way ANOVA with post-hoc Tukey's HSD test was used to assess significance $(p<0.05)$.

\section{Scanning electron microscopy (SEM)}

Flies were anaesthetised using FlyNap (Carolina Biologicals) and fixed in primary modified Karnovsky's fixative (3\% glutaraldehyde, $2 \%$ formaldehyde in $0.1 \mathrm{M}$ phosphate buffer, $\mathrm{pH} 7.2$ ) with Triton $\mathrm{X}-100$ by vacuum infiltration. They were then placed in fresh fixative and incubated at room temperature $>8$ hours, followed by $3 x$ 10 min washes in phosphate buffer $(0.1 \mathrm{M}, \mathrm{pH} 7.2)$. Dehydration was carried out via a graded ethanol series for ten to fifteen minutes at $25 \%, 50 \%, 75 \%, 95 \%$, and $100 \%$ ethanol, followed by a final one-hour incubation in $100 \%$ ethanol. The flies were then critical point dried using $\mathrm{CO}_{2}$ and $100 \%$ ethanol (Polaron E3000 series II drying apparatus). Heads were removed, mounted onto aluminium stubs and sputter coated with gold (Baltex SCD 050 sputter coater). Imaging was performed with a FEI Quanta 200 Environmental Scanning Electron Microscope at an accelerating voltage of $20 \mathrm{kV}$. To determine the sizes of each eye for comparison between genotypes, ImageJ software was used to draw a line surrounding the eye and calculate the area in 
arbitrary units. Statistical analysis was performed with one-way ANOVA with post-hoc Tukey's HSD test with significance set at $p<0.05$.

To provide a semi-quantitative analysis of SEM images, a scoring system was developed based on observations of phenotypes resulting from overexpression of one or two copies of UAS-HDAC4 in a previous study (SCHWARTZ et al. 2016). No defects: the eye appears wild-type - ommatidia are organised in a regular array with no fusion and mechanosensory bristles are correctly positioned between each ommatidium. Mild: Presence of one of the following phenotypes; between 5 - 10 instances of an abnormal number of interommatidial bristles, mild ommatidial disorganisation or fusion of ommatidia in up to two areas. Moderate: all mild phenotypes were collectively observed or one of the following phenotypes was observed; between 10 - 20 instances of an abnormal number of interommatidial bristles, moderate disorganisation or fusion of ommatidia in up to five areas. Major: all moderate phenotypes were collectively observed or one of the following phenotypes was observed; more than 20 instances of an abnormal number of interommatidial bristles, major disorganisation of the ommatidial array, fusion of ommatidia in up to 10 areas with few large areas of fusion or up to 50 collapsed ommatidia. Severe: all major phenotypes were collectively observed or one of the following phenotypes was observed; severe disorganisation, fusion in more than 10 areas or multiple large patches, more than 50 collapsed ommatidia or severe collapsing of ommatidia resulting in central hole-like cavities. Statistical analysis was assessed with the Fisher's Exact test with significance set at $p<0.05$.

\section{RESULTS}

\section{Characterisation of Ank2 expression in the brain}

To date, the expression and localisation pattern of Ank2 has been described in the Drosophila neuromuscular junction (KoCH et al. 2008; PIELAGE et al. 2008) however the expression pattern in the adult brain has not been characterized. Immunohistochemistry on whole mount brains with an antibody that detects Ank2-L (KoCH et al. 2008) indicated a broad expression profile with high expression in the optic lobes, antennal lobes, mushroom body and axon tracts throughout the brain (Fig 1A,B). Colocalization with the axonal marker Futsch (HUMmel et al. 2000) confirmed that Ank2-L localizes to axon tracts across the adult brain (Fig 1C). Ank2-L did not codistribute with the glial marker Reversed Polarity (Repo) (ALFONSO AND JONES 2002), confirming its specific neuronal expression pattern (Fig 1D). Since the mushroom body is a critical structure for memory (HEISENBERG et al. 1985; McBRIDE et al. 1999), we examined the expression and subcellular distribution of Ank2 in this brain area in more detail. The intrinsic neurons of the mushroom body are the Kenyon cells, which receive input from the olfactory system (TURNER et al. 2008). The cell bodies of the approximately 2,500 Kenyon cells are clustered in the posterior dorsal region of the brain and extend their dendrites anteriorly into a globular region known as the calyx, which is organized into an array of microglomeruli, each comprising the large synaptic bouton of projection neurons from the antennal lobe surrounded by Kenyon cell dendrites (LEISS et al. 2009). Their axons form a bundled fiber termed the pedunculus and project towards the anterior portion of the brain, forming five distinct lobes; the vertical $\alpha$ and $\alpha^{\prime}$ lobes and the medial $\beta, \beta^{\prime}$ and $\gamma$ lobes (CRITTENDEN et al. 1998; LEE AND 
Luo 1999) (see Fig. 3F). We examined the colocalization of Ank2-L with the neuronal cell adhesion molecule Neuroglian ( $\mathrm{Nrg}$ ), which has been shown to interact with Ank2 (ENNEKING et al. 2013; SIEGeNTHALER et al. 2015). Nrg is the sole Drosophila orthologue of the L1-CAM family of proteins (BIEBER et al. 1989), which enables axon guidance through the mushroom body. We confirmed that Ank2-L and Nrg codistributed in multiple axon tracts including the axons of the mushroom body, where both were observed in the $\alpha, \beta$ and $\gamma$ lobes (Fig 1E). Ank2-L was also concentrated in axon tracts surrounding the calyx of the mushroom body (Fig 1F).

\section{Ank2 is essential for axon and dendrite morphogenesis}

We previously found that overexpression of HDAC4 impairs morphogenesis of mushroom body axons, with deficits in axon branching and guidance observed as missing $\alpha$ and/or $\beta$ lobes, misdirected axons as well as the appearance of fused $\beta$ lobes, resulting from defects in axon termination across the midline (MAIN 2021). To investigate whether Ank2 is also required for axon morphogenesis, the morphology of the mushroom body in brains with reduced Ank2 was analyzed via detection of Fasciclin II. This cell adhesion molecule is highly expressed in the $\alpha, \beta$ and $\gamma$ lobes of the mushroom body (CRITTENDEN et al. 1998) and is a commonly used marker to visualise mushroom body lobe architecture (Fig 2A). Pan-neuronal knockdown of Ank2 with an inverted repeat hairpin that targets all long isoforms of Ank2 mRNA for degradation resulted in a variety of phenotypic defects of the mushroom body, including thin lobes, missing lobes and guidance abnormalities (Fig 2B-F). GAL4 activity increases at higher temperatures and accordingly we observed more severe defects when the temperature was raised during larval development (Table 1).

During Drosophila embryonic and larval stages, Ank2 mutants exhibit reduced dendritic branching, and in Drosophila dopaminergic neurons, knockdown of Ank2 results in decreased dendritic branching points, leading to a reduced total branch length and a lack of branching complexity (AVERY et al. 2017). To that end, we investigated whether Ank2 is required for dendrite morphogenesis in the adult Drosophila brain. As branching and elongation of Kenyon cell dendrites is difficult to visualize, we instead examined lobular plate tangential cells (LPTCS) of the visual system. The LPTCS are a group of six interneurons in the optic lobe that provide an ideal model system for investigating dendrite growth and branching as they display stereotypical dendritic arborization (LEISS et al. 2009). Individual dendrites are easily visualised via expression of Lifeact, a GFP-fused F-actin binding peptide (RIEDL et al. 2008) with the 3A-GAL4 driver (SCOTT et al. 2002) (Figure 2G), and branch length can be traced and quantified (Figure $2 \mathrm{H}, \mathrm{I}$ ). The characteristic arborization pattern of the six neurons was disrupted by expression of Ank2 RNAi with severely reduced dendritic projections (Figure $2 \mathrm{~J}, \mathrm{~K}$ ) leading to reduced total branch length (Figure $2 \mathrm{~L}$ ). These data suggest that wild-type levels of Ank2 are required for both axon branching, guidance and elongation was well as normal dendritic branching and arborization.

\section{LTM requires Ank2 expression in the $\gamma$ lobe of the mushroom body}

We next assessed whether Ank2 was required for memory formation with the repeat training courtship suppression assay. This test evaluates the memory of a male following exposure to an unreceptive mated female. Following this failure of mating, 
371 a male suppresses his courtship activity towards mated females to which he is 372 subsequently presented. After seven hours of training, males form a stable long-term 373 memory that lasts for at least 24 hours (KELEMAN et al. 2007; FITZSIMONS AND SCOTT 2011; 374 Fitzsimons et al. 2013). After this time, each male is placed with a new freshly mated 375 (unreceptive) female and a courtship index is calculated by dividing the amount of time each male spends courting by the total duration of the observation period. A memory index is calculated by comparing the time a trained male spends courting to that of a sham male. A score of zero indicates that memory is impaired and no different from untrained sham controls, whereas a higher memory index indicates intact memory. This form of courtship memory has been recently described as "cVAretrievable memory" to differentiate from the associative memory formed when virgin females are used for testing, which uses different circuitry for memory retrieval (RAUN et al. 2021). Learning and immediate short-term memory were unaffected by pan-neuronal knockdown of Ank2 (Fig 3A,B). Long-term courtship memory is dependent on an intact mushroom body, therefore unsurprisingly, pan-neuronal knockdown of Ank2 during development resulted in a significant and severe loss of LTM formation compared to control genotypes (Fig 3C). This was not due to an effect on courtship behavior as sham males of each genotype all spent approximately the same percentage of time courting ( 87 to $89 \%$, Figure 3D).

To avoid the developmental deficits resulting from decreased Ank2 expression and allow assessment of the role of Ank2 specifically in adult memory processes, knockdown of Ank2 was restricted to the mature brain with GAL80ts, a temperature sensitive inhibitor of GAL4 activity (MCGUIRE et al. 2004) (Fig 3E). Flies were raised at the permissive temperature of $19^{\circ} \mathrm{C}$ at which GAL80ts is active. Seventy-two hours after eclosion, male flies from the F1 progeny were collected individually and transferred to $30^{\circ} \mathrm{C}$ to inactivate GAL80ts and thus induce pan-neuronal RNAi expression. After three days, males were tested in the courtship suppression assay. Adult-specific knockdown of Ank2 in all neurons resulted in impairment of LTM formation (Fig 3G) and when knockdown of Ank2 was restricted to the adult mushroom body (Fig 3F) with OK107-GAL4, this impairment remained (Fig 3H). The three Kenyon cell subtypes are structurally distinct with individually identifiable transcriptomes (CROSET et al. 2018) and distinct roles in learning and memory (JOINER AND GRIFFITH 1999; KELEMAN et al. 2007), thus we next investigated whether there was a differential requirement for Ank2 in specific mushroom body subtypes by restricting expression of $A n k 2$ RNAi to each subtype individually (Fig 3F). Expression with the $\alpha / \beta$ and $\gamma$ neuron driver MB247-GAL4 abolished LTM formation (Fig 3I). Knockdown in the $\alpha / \beta$ neurons or $\alpha^{\prime} / \beta^{\prime}$ neurons did not significantly alter LTM (Fig 3J,K) whereas knockdown in $\gamma$ neurons with NP1131-GAL4 prevented LTM formation (Fig 3L). The $\gamma$ neuron driver 1471-GAL4 did not quite impair memory to significant levels (Fig 3M),

411 however this is a much weaker than NP1131-GAL4 (Aso et al. 2009). We therefore 412 tested an additional $\gamma$ neuron driver R16A06-GAL4 which drives very strong expression 413 in the gamma lobe, but minimal expression elsewhere in the brain (JENETT et al. 2012), 414 and employed a second independent Ank2 RNAi line that also targets all long forms of 415 Ank2 mRNA, which together resulted in a significant reduction in LTM (Fig 3N). 
417 Taken together these data show that Ank2- $L$ is required for normal mushroom body 418 development and in the adult brain, wild-type levels of Ank2-L are required in the $\gamma$ 419 lobes for normal LTM formation. This is strikingly similar to the phenotypes resulting 420 from manipulation of HDAC4 expression in that it is also required in the $\gamma$ lobe for LTM 421 but not STM (FITZSIMONS et al. 2013).

\section{HDAC4 does not physically interact with Ank2 in Drosophila neurons}

The $\mathrm{N}$-terminal region of HDAC4 contains an ankyrin repeat binding domain consisting of a PxLPxI/L motif (Fig 4A), which in mammalian cells binds the ankyrin repeat region of ANKRA2 and RFXANK (WANG et al. 2005; Xu et al. 2012; NIE et al. 2015b). This motif is conserved in Drosophila HDAC4, and the ankyrin repeat of RFXANK and ANKRA2 both share $52 \%$ amino acid similarity with that of Ank2, thus it is possible that Ank2 and HDAC4 could interact physically through the ankyrin repeat of Ank2. To test for a physical interaction, we generated flies that co-express the ankyrin repeat-containing domain of Ank2 with a C-terminal HA tag (UAS-Ank2 ${ }_{190-946-H A)}$ and Myc-tagged Drosophila HDAC4 in the brain. Coimmunoprecipitation on head lysates revealed no detectable interaction of HDAC4 with Ank2 via pulldown with either anti-HA or antiMyc (Fig 4B,C), therefore while Ank2 and HDAC4 interact genetically, they do not appear to physically interact at a detectable level in the brain. An obvious explanation for the genetic interaction between the two would be that HDAC4 regulates expression of $A n k 2$, however previous RNA-seq data in which we expressed DmHDAC4 or a nuclear-restricted mutant of human HDAC4 did not show a significant change in transcription of Ank2 (SCHWARTZ et al. 2016; MAIN 2021). We also confirmed that increased HDAC4 expression does not alter the level of Ank2 protein (Fig 4D,E).

\section{Nuclear HDAC4 mediates the genetic interaction with Ank2}

We previously showed that knockdown of Ank2 in the eye resulted in a mild developmental impairment that was significantly enhanced when combined with HDAC4 overexpression, indicating the two genes interact in the same molecular pathway (SCHWARTZ et al. 2016). The HDAC4-induced impairments in eye development are largely a consequence of nuclear accumulation of HDAC4, as expression of a mutant variant of human HDAC4 that is sequestered in the nucleus resulted in a more severe phenotype than overexpression of wild-type human HDAC4, whereas the phenotype resulting from expression of a cytoplasm-restricted mutant was mild (MAIN 2021). To that end, we next investigated whether the genetic interaction between Ank2 and HDAC4 in the eye is also dependent on the nuclear presence of Drosophila HDAC4. In addition, we sought to determine whether the genetic interaction is dependent on the putative ankyrin repeat-binding motif region of HDAC4. If not, this would provide further confirmation that the genetic interaction between HDAC4 and Ank2 is not through direct physical binding.

We first confirmed our previous observations by co-expressing DmHDAC4 and Ank2 RNAi under the control of GMR-GAL4, which drives expression in post-mitotic cells posterior to the morphogenetic furrow (FREEMAN 1996), and examining the phenotype of adult eyes. We also raised the temperature to $27^{\circ} \mathrm{C}$ to increase GAL4 activity and thus increase the degree of knockdown in order to examine the resulting phenotypic defects in eye development in more detail. Control flies (GMR-GAL4 crossed to the 
background $w[C S 10]$ strain) showed predominantly normal ommatidial alignment and no evidence of fusion (Fig 5A), whereas eyes with reduced Ank2 expression had fused, collapsed and misaligned ommatidia lacking some interommatidial bristles (Fig 5B). Overexpression of DmHDAC4 also resulted in missing/disorganised bristles and misaligned and fused ommatidia (Fig 5C). Combined expression of DmHDAC4 and Ank2 RNAi resulted in a more severe rough eye phenotype consisting of major areas of ommatidial fusion, severe misalignment with hole-like cavities within the ommatidia, and missing/disorganised bristles (Fig 5D). The severity of phenotypes was scored (Table S1) and the percentage of eyes displaying the most severe phenotype was significantly higher when DmHDAC4 and Ank2 RNAi were combined (Fig 5I). In addition to the severe eye phenotypes observed above, the eyes were also significantly physically smaller than those expressing either Ank2 RNAi or DmHDAC4 individually (Fig 5J). Together these synergistic phenotypes are consistent with our previous findings (SCHWARTZ et al. 2016) and provide further confirmation that Ank2 and HDAC4 genetically interact together to influence eye development.

To further investigate the mechanism of this interaction, we examined whether it was dependent on the nuclear activity of HDAC4. A nuclear-restricted mutant of HDAC4 (DmHDAC4 3SA) resulted in a more severe phenotype than wild-type HDAC4, with increased disorganisation and fusion of ommatidia and a reduced number of bristles (Fig 5E), which we have previously observed (MAIN 2021). DmHDAC4 3 SA interacted synergistically with Ank2 (Fig 5F), confirming that nuclear activity of HDAC4 is required for the genetic interaction. To address whether the putative ankyrin binding motif in Drosophila HDAC4 is required we substituted residues that have been shown to be important for this interaction in mammals; P48 L50 P51 and I53 of the PxLPxl/L motif (Fig 4A) to alanines to create the DmHDAC4 $\triangle A n k$ mutant. Expression of this mutant also resulted in a moderate rough eye phenotype consisting of ommatidial fusion and misalignment (Figure $5 \mathrm{G}$ ), indicating that the presence of this motif is not required for the HDAC4 overexpression-induced eye defects. This mutant still interacted genetically with $A n k 2$, resulting in a significantly more severe rough eye phenotype and reduced eye size when expressed in combination with Ank2 (Fig 5H), therefore this interaction does not depend on the presence of the putative ankyrin-binding motif of HDAC4, which is consistent with the lack of physical interaction with Ank2.

\section{DISCUSSION}

Here, we provide the first characterization of the expression pattern of Ank2 in the adult Drosophila brain and demonstrate that Ank2 is critical for normal development of the mushroom body and for formation of long-term memories.

Ank2 expression was observed throughout the brain and localized predominantly to axon tracts, including the lobes of the mushroom body where it colocalized with $\mathrm{Nrg}$. As the mushroom body is a critical structure for memory formation (HEISENBERG et al. 1985; MCBRIDE et al. 1999), and we previously showed that HDAC4 is required in the mushroom body for normal memory formation, and that overexpression of HDAC4 impairs mushroom body development (FITZSIMONS et al. 2013; MAIN 2021), we focused our attention on examining the role of Ank2 in both developmental and postdevelopmental processes in this brain region. Pan-neuronal knockdown of Ank2 in the 
511 developing mushroom body resulted in deficits in axon guidance and elongation. Ank2 512 has been demonstrated to interact with Nrg through a FIGQY motif within the 513 intracellular domain of $\mathrm{Nrg}$ (ENNEKING et al. 2013). Nrg is required for normal 514 mushroom body lobe development, whereby the extracellular domain of $\mathrm{Nrg}$ 515 mediates cellular adhesion between axons of different mushroom body subtypes for 516 guidance into the pedunculus and lobes. This interaction relies on the presence of 517 Ank2 in either the ingrowing or substrate axon neurons where it is proposed to 518 stabilise the transaxonal Nrg complex (SIEGENTHALER et al. 2015). A hypomorphic Nrg 519 mutant displayed deficits in axon growth and guidance in the mushroom body 520 (SIEGENTHALER et al. 2015), which are very similar to those we observed on knockdown 521 of Ank2. In addition, Nrg also interacts with a second cytoskeletal adapter protein 522 Moesin (Moe) through a FERM domain in the intracellular domain of L1 CAMs (DICKSON 523 et al. 2002), creating a ternary complex between Ank2, Nrg and Moe (SIEGENTHALER et 524 al. 2015). Moe is highly expressed in the mushroom body and distributes to the lobes on activation by phosphorylation (FREYMUTH AND FITZSIMONS 2017), and likely acts to link the Ank2-Nrg complex to the actin cytoskeleton in mushroom body axons. Interestingly we also previously showed that HDAC4 interacts genetically with Moe (SCHWARTZ et al. 2016), and moreover, reduction of Moe also shows similar disruption to mushroom body development as with Ank2 and $\mathrm{Nrg}$, with defects in axon elongation and guidance (SIEgenthaler et al. 2015; FreYMUTH AND FITZSIMONS 2017). Together these data support the evidence for a functional relationship between Ank2, $\mathrm{Nrg}$ and Moe in mushroom body development.

Pan-neuronal knockdown of Ank2 also severely impaired 24-hour LTM without affecting courtship behavior, learning, or immediate courtship memory. It was previously found that decreased expression of Ank2 in the mushroom body with OK107-GAL4 did not result in learning deficits but caused significant impairment to one-hour STM in the same assay (IQBAL et al. 2013). Similarly, pan-neuronal knockdown of Ank2 with elav-GAL4 impaired one hour olfactory memory (HIGHAM et al. 2019) and mushroom body-specific knockdown with nSyb-GAL4 impaired threehour memory when tested in the olfactory conditioning assay (WALKINSHAW et al. 2015). However, in these studies the drivers are expressed in larvae at which time the mushroom body is developing (Nicolal et al. 2003; OGIENKO et al. 2020; KOBLER et al. 2021), and an intact mushroom body is required for normal olfactory STM (HEISENBERG et al. 1985) and associative STM lasting longer than 30 mins (MCBRIDE et al. 1999). Furthermore, output from $\gamma$ neurons is required for short-term courtship memory (KELEMAN et al. 2012) and knockdown of Ank2 in $y$ neurons during mushroom body development was recently found to result in a shortened axon initial segment in third instar larval brains (SPURRIER et al. 2019). In light of the severe mushroom body defects we observed on knockdown of $A n k 2$, and that Ank2 has been implicated in synapse stability (HORTSCH et al. 2002; KOCH et al. 2008), it is unsurprising that memory would be impaired, and from these data it cannot be determined whether Ank2 plays a specific role in memory or whether the defects are a result of impaired morphogenesis of the mushroom body. To that end, in order to dissociate developmental effects from the molecular processes required for LTM in an adult brain, knockdown of Ank2 was restricted to mature neurons with GAL80. A specific deficit in 24 hour LTM was

557 observed when Ank2 was knocked down in the adult mushroom body, and subsequent 
testing of GAL4 drivers that restrict expression to specific mushroom body subtypes revealed that knockdown in just the $y$ neurons was sufficient to impair memory. We previously showed that overexpression of HDAC4 in $\gamma$ neurons also impaired the formation of long-term courtship memory (SCHWARTZ et al. 2016) and strikingly, in accordance with the similarity in mushroom body defects, knockdown of Moe resulted in the same phenotype as Ank2 knockdown and HDAC4 overexpression (FITZsIMONs et al. 2013; FREYMUTH AND FITZSIMONS 2017), suggesting that a functional relationship between these proteins also occurs during LTM formation.

The identification that Ank2 is required in the $\gamma$ neurons of the mushroom body for normal cVA-retrievable memory is consistent with current models of the circuitry that facilitates this memory (KELEMAN et al. 2012; RAUN et al. 2021). cVA-retrievable memory involves activation of aSP13 dopaminergic neurons which innervate the $\gamma 5$ compartment at tip of the $\gamma$ lobe. This results in increased synaptic transmission from $\gamma$ neurons to glutamatergic M6 output neurons, which themselves feedback to innervate aSP13 neurons to form a recurrent activation loop (KELEMAN et al. 2012; ZHAO et al. 2018). Long-term memory requires a later reactivation of aSP13 neurons, which is dependent on sleep (DAG et al. 2019). Expression of epigenetic regulators required for cVA-retrievable memory, including Rpd3, HDAC4 and G9a has also been pinpointed to a requirement in only $\gamma$ neurons. Similarly, the cytoplasmic polyadenylation element-binding protein Orb2 is required for translation of synaptic mRNA during LTM consolidation in the y lobe (KRUTTNER et al. 2015).

Although Ank2 and HDAC4 both localize to axons of the mushroom body, and overexpression of HDAC4 in the mushroom body also results in impaired axon elongation and guidance defects as well as deficits in LTM formation (FITZSIMONS et al. 2013; FREYMUTH AND FITZSIMONS 2017; MAIN 2021), we found no evidence of a physical interaction in Drosophila neurons. We further investigated the interaction between HDAC4 and Ank2 in the eye and observed that a nuclear-restricted mutant of HDAC4 interacted genetically with $A n k 2$, however this was not dependent on the presence of a conserved putative ankyrin-binding motif in HDAC4. Given the striking similarity of the Ank2 and Moe knockdown phenotypes with HDAC4 overexpression, it is possible that when increased in abundance in Kenyon cell nuclei, HDAC4 may act indirectly to disrupt the normal role of the Ank2, Nrg, and Moe complex. It is not yet known how an HDAC4-mediated signal from the nucleus modulates these or other proteins involved in neuronal morphogenesis and LTM. We previously found that HDAC4 also interacts genetically with several components of the SUMOylation machinery, including the SUMO E2-conjugating enzyme Ubc9, which is also required for long-term courtship memory and interacts genetically with HDAC4 during this process (SCHWARTZ et al. 2016). HDAC4 has been proposed to act as an E3 ligase to enhance SUMOylation of target proteins (GREGOIRE AND YANG 2005; ZHAO et al. 2005). Given that SUMOylation regulates neuronal protein activity (HENLEY et al. 2014) and regulates memory formation (YANG et al. 2012; CHEN et al. 2014; LEE et al. 2014; Yu et al. 2020), it would be worthwhile to further investigate whether any of the HDAC4-interacting genes are SUMOylated and whether this is impacted by altering the expression level or subcellular distribution of HDAC4. 
In our screen for genes that enhanced the HDAC4-induced rough eye phenotype (SCHWARTZ et al. 2016), the largest group of genes we identified composed of regulators of the actin/spectrin cytoskeleton such as Trio, NetrinB, Derailed, Ankyrin, Ankyrin2, and Moesin, suggesting that HDAC4 might regulate memory through interaction with genes involved in remodelling of the actin/spectrin cytoskeleton. This is a phenomenon which is believed to regulate the structural changes including polymerization/depolymerization or alterations of the underlying actin cytoskeleton that underpin learning and memory (ENGERT AND BONHOEFFER 1999; KRUCKER et al. 2000; LAMPRECHT AND LEDOUX 2004; LeISS et al. 2009; OJELADE et al. 2013; LAMPRECHT 2014). We previously showed that expression of a constitutively active mutant of Moe increases the density of F-actin in spine-like structures in LPTC neurons (FREYMUTH AND FITZSIMONS 2017). In mammals, AnkG is present at synapses and is required for formation of nanodomain structures in the perisynaptic spine and spine neck of dendrites, the contents of which include adhesion molecules, F-actin and CaMKII, thus facilitating interaction with the actin cytoskeleton for F-actin rearrangements that underlie dynamic alterations in spine morphology. The geometry of the spine in this region increases on induction of long-term potentiation in primary cortical neurons, and reduction of AnkG prevents spine enlargement (SMITH et al. 2014). It is also notable that inhibition of actin polymerization within the mushroom body of the honeybee enhanced associative olfactory memory (GANESHINA et al. 2012). As reduced HDAC4 also impairs LTM, indicating an essential role in memory formation, the impact of both increased and reduced HDAC4 on cytoskeletal rearrangement warrants further investigation.

\section{Acknowledgements}

We thank Hermann Aberle (Heinrich-Heine-Universtität, Düsseldorf, Germany) for the Ank2-L antibody and Max Scott for constructive comments on the manuscript. We also thank the Manawatu Microscopy and Imaging Centre, Massey University for assistance with confocal and SEM.

\section{Funding}

This work was supported by the Royal Society of New Zealand (Marsden grant MAU1702 to HLF) and the Massey University Research Fund.

\section{References}

Alfonso, T. B., and B. W. Jones, $2002 \mathrm{gcm} 2$ promotes glial cell differentiation and is required with glial cells missing for macrophage development in Drosophila. Dev Biol 248: 369-383.

Aso, Y., K. Grubel, S. Busch, A. B. Friedrich, I. Siwanowicz et al., 2009 The mushroom body of adult Drosophila characterized by GAL4 drivers. J Neurogenet 23: 156172.

Athanasiu, L., M. Mattingsdal, A. K. Kahler, A. Brown, O. Gustafsson et al., 2010 Gene variants associated with schizophrenia in a Norwegian genome-wide study are replicated in a large European cohort. Journal of psychiatric research 44: 748753. 
Avery, A. W., D. D. Thomas and T. S. Hays, 2017 beta-III-spectrin spinocerebellar ataxia type 5 mutation reveals a dominant cytoskeletal mechanism that underlies dendritic arborization. Proc Natl Acad Sci U S A 114: E9376-E9385.

Bennett, V., 1978 Purification of an active proteolytic fragment of the membrane attachment site for human erythrocyte spectrin. J Biol Chem 253: 2292-2299.

Bennett, V., and A. J. Baines, 2001 Spectrin and ankyrin-based pathways: metazoan inventions for integrating cells into tissues. Physiol Rev 81: 1353-1392.

Bi, C., J. Wu, T. Jiang, Q. Liu, W. Cai et al., 2012 Mutations of ANK3 identified by exome sequencing are associated with autism susceptibility. Hum Mutat 33: 16351638.

Bieber, A. J., P. M. Snow, M. Hortsch, N. H. Patel, J. R. Jacobs et al., 1989 Drosophila neuroglian: a member of the immunoglobulin superfamily with extensive homology to the vertebrate neural adhesion molecule L1. Cell 59: 447-460.

Bottomley, M. J., P. Lo Surdo, P. Di Giovine, A. Cirillo, R. Scarpelli et al., 2008 Structural and functional analysis of the human HDAC4 catalytic domain reveals a regulatory structural zinc-binding domain. J Biol Chem 283: 26694-26704.

Chawla, S., P. Vanhoutte, F. J. Arnold, C. L. Huang and H. Bading, 2003 Neuronal activity-dependent nucleocytoplasmic shuttling of HDAC4 and HDAC5. Journal of neurochemistry 85: 151-159.

Chen, Y. C., W. L. Hsu, Y. L. Ma, D. J. Tai and E. H. Lee, 2014 CREB SUMOylation by the E3 ligase PIAS1 enhances spatial memory. The Journal of neuroscience : the official journal of the Society for Neuroscience 34: 9574-9589.

Crittenden, J. R., E. M. Skoulakis, K. A. Han, D. Kalderon and R. L. Davis, 1998 Tripartite mushroom body architecture revealed by antigenic markers. Learn Mem 5: 3851.

Croset, V., C. D. Treiber and S. Waddell, 2018 Cellular diversity in the Drosophila midbrain revealed by single-cell transcriptomics. Elife 7.

Cunha, S. R., and P. J. Mohler, 2009 Ankyrin protein networks in membrane formation and stabilization. J Cell Mol Med 13: 4364-4376.

Dag, U., Z. Lei, J. Q. Le, A. Wong, D. Bushey et al., 2019 Neuronal reactivation during post-learning sleep consolidates long-term memory in Drosophila. Elife 8.

Dickson, T. C., C. D. Mintz, D. L. Benson and S. R. Salton, 2002 Functional binding interaction identified between the axonal CAM L1 and members of the ERM family. J Cell Biol 157: 1105-1112.

Ejima, A., B. P. Smith, C. Lucas, J. D. Levine and L. C. Griffith, 2005 Sequential learning of pheromonal cues modulates memory consolidation in trainer-specific associative courtship conditioning. Current biology : CB 15: 194-206.

Engert, F., and T. Bonhoeffer, 1999 Dendritic spine changes associated with hippocampal long-term synaptic plasticity. Nature 399: 66-70.

Enneking, E. M., S. R. Kudumala, E. Moreno, R. Stephan, J. Boerner et al., 2013 Transsynaptic coordination of synaptic growth, function, and stability by the L1-type CAM Neuroglian. PLoS Biol 11: e1001537.

Fitzsimons, H. L., 2015 The Class Ila histone deacetylase HDAC4 and neuronal function: Nuclear nuisance and cytoplasmic stalwart? Neurobiol Learn Mem 123: 149158.

Fitzsimons, H. L., S. Schwartz, F. M. Given and M. J. Scott, 2013 The histone deacetylase HDAC4 regulates long-term memory in Drosophila. PloS one 8: e83903. 
Fitzsimons, H. L., and M. J. Scott, 2011 Genetic modulation of Rpd3 expression impairs long-term courtship memory in Drosophila. PloS one 6: e29171.

Freeman, M., 1996 Reiterative use of the EGF receptor triggers differentiation of all cell types in the Drosophila eye. Cell 87: 651-660.

Freymuth, P. S., and H. L. Fitzsimons, 2017 The ERM protein Moesin is essential for neuronal morphogenesis and long-term memory in Drosophila. Mol Brain 10: 41.

Ganeshina, O., J. Erdmann, S. Tiberi, M. Vorobyev and R. Menzel, 2012 Depolymerization of actin facilitates memory formation in an insect. Biol Lett 8: 1023-1027.

Gregoire, S., and X. J. Yang, 2005 Association with class Ila histone deacetylases upregulates the sumoylation of MEF2 transcription factors. Molecular and cellular biology 25: 2273-2287.

Grozinger, C. M., and S. L. Schreiber, 2000 Regulation of histone deacetylase 4 and 5 and transcriptional activity by 14-3-3-dependent cellular localization. Proc Natl Acad Sci U S A 97: 7835-7840.

Guo, X., Y. Zhang, J. Du, H. Yang, Y. Ma et al., 2016 Association analysis of ANK3 gene variants with schizophrenia in a northern Chinese Han population. Oncotarget 7: 85888-85894.

Heisenberg, M., A. Borst, S. Wagner and D. Byers, 1985 Drosophila mushroom body mutants are deficient in olfactory learning. J Neurogenet 2: 1-30.

Henley, J. M., T. J. Craig and K. A. Wilkinson, 2014 Neuronal SUMOylation: mechanisms, physiology, and roles in neuronal dysfunction. Physiological reviews 94: 1249-1285.

Herrup, K., J. Li and J. Chen, 2013 The role of ATM and DNA damage in neurons: upstream and downstream connections. DNA repair 12: 600-604.

Higham, J. P., B. R. Malik, E. Buhl, J. M. Dawson, A. S. Ogier et al., 2019 Alzheimer's Disease Associated Genes Ankyrin and Tau Cause Shortened Lifespan and Memory Loss in Drosophila. Front Cell Neurosci 13: 260.

Hortsch, M., K. L. Paisley, M. Z. Tian, M. Qian, M. Bouley et al., 2002 The axonal localization of large Drosophila ankyrin2 protein isoforms is essential for neuronal functionality. Mol Cell Neurosci 20: 43-55.

Hughes, T., I. E. Sonderby, T. Polushina, L. Hansson, A. Holmgren et al., 2018 Elevated expression of a minor isoform of ANK3 is a risk factor for bipolar disorder. Transl Psychiatry 8: 210.

Hummel, T., K. Krukkert, J. Roos, G. Davis and C. Klambt, 2000 Drosophila Futsch/22C10 is a MAP1B-like protein required for dendritic and axonal development. Neuron 26: 357-370.

Iqbal, Z., G. Vandeweyer, M. van der Voet, A. M. Waryah, M. Y. Zahoor et al., 2013 Homozygous and heterozygous disruptions of ANK3: at the crossroads of neurodevelopmental and psychiatric disorders. Human molecular genetics 22: 1960-1970.

Jenett, A., G. M. Rubin, T. T. Ngo, D. Shepherd, C. Murphy et al., 2012 A GAL4-driver line resource for Drosophila neurobiology. Cell Rep 2: 991-1001.

Joiner, M. A., and L. C. Griffith, 1999 Mapping of the anatomical circuit of CaM kinasedependent courtship conditioning in Drosophila. Learn Mem 6: 177-192. 
Keleman, K., S. Kruttner, M. Alenius and B. J. Dickson, 2007 Function of the Drosophila CPEB protein Orb2 in long-term courtship memory. Nat Neurosci 10: 15871593.

Keleman, K., E. Vrontou, S. Kruttner, J. Y. Yu, A. Kurtovic-Kozaric et al., 2012 Dopamine neurons modulate pheromone responses in Drosophila courtship learning. Nature 489: 145-149.

Kim, M. S., M. W. Akhtar, M. Adachi, M. Mahgoub, R. Bassel-Duby et al., 2012a An essential role for histone deacetylase 4 in synaptic plasticity and memory formation. The Journal of neuroscience : the official journal of the Society for Neuroscience 32: 10879-10886.

Kim, M. S., M. W. Akhtar, M. Adachi, M. Mahgoub, R. Bassel-Duby et al., 2012b An essential role for histone deacetylase 4 in synaptic plasticity and memory formation. J Neurosci 32: 10879-10886.

Kobler, O., A. Weiglein, K. Hartung, Y. C. Chen, B. Gerber et al., 2021 A quick and versatile protocol for the $3 \mathrm{D}$ visualization of transgene expression across the whole body of larval Drosophila. J Neurogenet: 1-14.

Koch, I., H. Schwarz, D. Beuchle, B. Goellner, M. Langegger et al., 2008 Drosophila ankyrin 2 is required for synaptic stability. Neuron 58: 210-222.

Krucker, T., G. R. Siggins and S. Halpain, 2000 Dynamic actin filaments are required for stable long-term potentiation (LTP) in area CA1 of the hippocampus. Proc Natl Acad Sci U S A 97: 6856-6861.

Kruttner, S., L. Traunmuller, U. Dag, K. Jandrasits, B. Stepien et al., 2015 Synaptic Orb2A Bridges Memory Acquisition and Late Memory Consolidation in Drosophila. Cell Rep 11: 1953-1965.

Lahm, A., C. Paolini, M. Pallaoro, M. C. Nardi, P. Jones et al., 2007 Unraveling the hidden catalytic activity of vertebrate class Ila histone deacetylases. Proc Natl Acad Sci U S A 104: 17335-17340.

Lamprecht, R., 2014 The actin cytoskeleton in memory formation. Prog Neurobiol 117: 1-19.

Lamprecht, R., and J. LeDoux, 2004 Structural plasticity and memory. Nature reviews. Neuroscience 5: 45-54.

Lee, L., E. Dale, A. Staniszewski, H. Zhang, F. Saeed et al., 2014 Regulation of synaptic plasticity and cognition by SUMO in normal physiology and Alzheimer's disease. Sci Rep 4: 7190.

Lee, T., and L. Luo, 1999 Mosaic analysis with a repressible cell marker for studies of gene function in neuronal morphogenesis. Neuron 22: 451-461.

Leiss, F., E. Koper, I. Hein, W. Fouquet, J. Lindner et al., 2009 Characterization of dendritic spines in the Drosophila central nervous system. Developmental neurobiology 69: 221-234.

Li, J., J. Chen, C. L. Ricupero, R. P. Hart, M. S. Schwartz et al., 2012 Nuclear accumulation of HDAC4 in ATM deficiency promotes neurodegeneration in ataxia telangiectasia. Nature medicine 18: 783-790.

Livak, K. J., and T. D. Schmittgen, 2001 Analysis of relative gene expression data using real-time quantitative PCR and the 2(-Delta Delta $C(T)$ ) Method. Methods 25: 402-408.

Main, P., Tan, W.J. Wheeler, D, Fitzsimons H.L., 2021 Increased abundance of nuclear HDAC4 impairs neuronal development and long-term memory. bioRxiv. 
McBride, S. M., G. Giuliani, C. Choi, P. Krause, D. Correale et al., 1999 Mushroom body ablation impairs short-term memory and long-term memory of courtship conditioning in Drosophila melanogaster. Neuron 24: 967-977.

McGuire, S. E., Z. Mao and R. L. Davis, 2004 Spatiotemporal gene expression targeting with the TARGET and gene-switch systems in Drosophila. Sci STKE 2004: pl6.

McKinsey, T. A., C. L. Zhang, J. Lu and E. N. Olson, 2000 Signal-dependent nuclear export of a histone deacetylase regulates muscle differentiation. Nature 408: 106-111.

Mielcarek, M., C. Landles, A. Weiss, A. Bradaia, T. Seredenina et al., 2013 HDAC4 reduction: a novel therapeutic strategy to target cytoplasmic huntingtin and ameliorate neurodegeneration. PLoS biology 11: e1001717.

Mohler, P. J., A. O. Gramolini and V. Bennett, 2002 The ankyrin-B C-terminal domain determines activity of ankyrin-B/G chimeras in rescue of abnormal inositol 1,4,5-trisphosphate and ryanodine receptor distribution in ankyrin-B (-/-) neonatal cardiomyocytes. J Biol Chem 277: 10599-10607.

Morgan, A. R., G. Hamilton, D. Turic, L. Jehu, D. Harold et al., 2008 Association analysis of 528 intra-genic SNPs in a region of chromosome 10 linked to late onset Alzheimer's disease. Am J Med Genet B Neuropsychiatr Genet 147B: 727-731.

Morris, B., C. Etoubleau, S. Bourthoumieu, S. Reynaud-Perrine, C. Laroche et al., 2012a Dose dependent expression of HDAC4 causes variable expressivity in a novel inherited case of brachydactyly mental retardation syndrome. American journal of medical genetics. Part A 158A: 2015-2020.

Nicolai, M., C. Lasbleiz and J. M. Dura, 2003 Gain-of-function screen identifies a role of the Src64 oncogene in Drosophila mushroom body development. J Neurobiol 57: 291-302.

Nie, F., X. Wang, P. Zhao, H. Yang, W. Zhu et al., 2015a Genetic analysis of SNPs in CACNA1C and ANK3 gene with schizophrenia: A comprehensive meta-analysis. Am J Med Genet B Neuropsychiatr Genet 168: 637-648.

Nie, J., C. Xu, J. Jin, J. A. Aka, W. Tempel et al., 2015b Ankyrin repeats of ANKRA2 recognize a PXLPXL motif on the $3 \mathrm{M}$ syndrome protein CCDC8. Structure 23: 700-712.

Ogienko, A. A., E. N. Andreyeva, E. S. Omelina, A. L. Oshchepkova and A. V. Pindyurin, 2020 Molecular and cytological analysis of widely-used Gal4 driver lines for Drosophila neurobiology. BMC Genet 21: 96.

Ojelade, S. A., S. F. Acevedo and A. Rothenfluh, 2013 The role of the actin cytoskeleton in regulating Drosophila behavior. Rev Neurosci 24: 471-484.

Pielage, J., L. Cheng, R. D. Fetter, P. M. Carlton, J. W. Sedat et al., 2008 A presynaptic giant ankyrin stabilizes the NMJ through regulation of presynaptic microtubules and transsynaptic cell adhesion. Neuron 58: 195-209.

Raun, N., S. Jones and J. M. Kramer, 2021 Conditioned courtship suppression in Drosophila melanogaster. J Neurogenet: 1-27.

Riedl, J., A. H. Crevenna, K. Kessenbrock, J. H. Yu, D. Neukirchen et al., 2008 Lifeact: a versatile marker to visualize F-actin. Nat Methods 5: 605-607.

Sando, R., 3rd, N. Gounko, S. Pieraut, L. Liao, J. Yates, 3rd et al., 2012 HDAC4 governs a transcriptional program essential for synaptic plasticity and memory. Cell 151: 821-834. 
Schlumm, F., D. Mauceri, H. E. Freitag and H. Bading, 2013 Nuclear calcium signaling regulates nuclear export of a subset of class Ila histone deacetylases following synaptic activity. J Biol Chem 288: 8074-8084.

Schwartz, S., M. Truglio, M. J. Scott and H. L. Fitzsimons, 2016 Long-Term Memory in Drosophila Is Influenced by Histone Deacetylase HDAC4 Interacting with SUMO-Conjugating Enzyme Ubc9. Genetics 203: 1249-1264.

Scott, E. K., T. Raabe and L. Luo, 2002 Structure of the vertical and horizontal system neurons of the lobula plate in Drosophila. J Comp Neurol 454: 470-481.

Sedgwick, S. G., and S. J. Smerdon, 1999 The ankyrin repeat: a diversity of interactions on a common structural framework. Trends Biochem Sci 24: 311-316.

Shen, X., J. Chen, J. Li, J. Kofler and K. Herrup, 2016 Neurons in Vulnerable Regions of the Alzheimer's Disease Brain Display Reduced ATM Signaling. eNeuro 3.

Siegenthaler, D., E. M. Enneking, E. Moreno and J. Pielage, 2015 L1CAM/Neuroglian controls the axon-axon interactions establishing layered and lobular mushroom body architecture. The Journal of cell biology 208: 1003-1018.

Smith, K. R., K. J. Kopeikina, J. M. Fawcett-Patel, K. Leaderbrand, R. Gao et al., 2014 Psychiatric risk factor ANK3/ankyrin-G nanodomains regulate the structure and function of glutamatergic synapses. Neuron 84: 399-415.

Spurrier, J., A. K. Shukla, T. Buckley, S. Smith-Trunova, I. Kuzina et al., 2019 Expression of a Fragment of Ankyrin 2 Disrupts the Structure of the Axon Initial Segment and Causes Axonal Degeneration in Drosophila. Mol Neurobiol 56: 5689-5700.

Tesli, M., P. Koefoed, L. Athanasiu, M. Mattingsdal, O. Gustafsson et al., 2011 Association analysis of ANK3 gene variants in nordic bipolar disorder and schizophrenia case-control samples. Am J Med Genet B Neuropsychiatr Genet 156B: 969-974.

Trazzi, S., C. Fuchs, R. Viggiano, M. De Franceschi, E. Valli et al., 2016 HDAC4: a key factor underlying brain developmental alterations in CDKL5 disorder. Hum Mol Genet 25: 3887-3907.

Turner, G. C., M. Bazhenov and G. Laurent, 2008 Olfactory representations by Drosophila mushroom body neurons. J Neurophysiol 99: 734-746.

Villavicencio-Lorini, P., E. Klopocki, M. Trimborn, R. Koll, S. Mundlos et al., 2013 Phenotypic variant of Brachydactyly-mental retardation syndrome in a family with an inherited interstitial 2q37.3 microdeletion including HDAC4. European journal of human genetics : EJHG 21: 743-748.

Wakeling, E., M. McEntagart, M. Bruccoleri, C. Shaw-Smith, K. L. Stals et al., 2021 Missense substitutions at a conserved 14-3-3 binding site in HDAC4 cause a novel intellectual disability syndrome. HGG Adv 2: 100015.

Walkinshaw, E., Y. Gai, C. Farkas, D. Richter, E. Nicholas et al., 2015 Identification of genes that promote or inhibit olfactory memory formation in Drosophila. Genetics 199: 1173-1182.

Wang, A. H., S. Gregoire, E. Zika, L. Xiao, C. S. Li et al., 2005 Identification of the ankyrin repeat proteins ANKRA and RFXANK as novel partners of class Ila histone deacetylases. J Biol Chem 280: 29117-29127.

Wang, A. H., M. J. Kruhlak, J. Wu, N. R. Bertos, M. Vezmar et al., 2000 Regulation of histone deacetylase 4 by binding of 14-3-3 proteins. Molecular and cellular biology 20: 6904-6912. 
882

883

884

885

886

887

888

889

890

891

892

893

894

895

896

897

898

899

900

901

902

903

904

905

906

907

908

909

910

911

912

913

914

915

916

917

918

919

920

921

922

Wang, A. H., and X. J. Yang, 2001 Histone deacetylase 4 possesses intrinsic nuclear import and export signals. Molecular and cellular biology 21: 5992-6005.

Wang, W. H., L. C. Cheng, F. Y. Pan, B. Xue, D. Y. Wang et al., 2011 Intracellular trafficking of histone deacetylase 4 regulates long-term memory formation. Anatomical record 294: 1025-1034.

Wheeler, P. G., D. Huang and Z. Dai, 2014 Haploinsufficiency of HDAC4 does not cause intellectual disability in all affected individuals. American journal of medical genetics. Part A 164A: 1826-1829.

Williams, S. R., M. A. Aldred, V. M. Der Kaloustian, F. Halal, G. Gowans et al., 2010a Haploinsufficiency of HDAC4 causes brachydactyly mental retardation syndrome, with brachydactyly type E, developmental delays, and behavioral problems. Am J Hum Genet 87: 219-228.

Xu, C., J. Jin, C. Bian, R. Lam, R. Tian et al., 2012 Sequence-specific recognition of a $\mathrm{PxLPxI} / \mathrm{L}$ motif by an ankyrin repeat tumbler lock. Sci Signal 5: ra39.

Yang, Q. G., F. Wang, Q. Zhang, W. R. Xu, Y. P. Chen et al., 2012 Correlation of increased hippocampal Sumo3 with spatial learning ability in old C57BL/6 mice. Neuroscience letters 518: 75-79.

Yu, S., F. Galeffi, R. M. Rodriguiz, Z. Wang, Y. Shen et al., 2020 Small ubiquitin-like modifier 2 (SUMO2) is critical for memory processes in mice. FASEB J 34: $14750-14767$.

Yuan, A., Z. Yi, Q. Wang, J. Sun, Z. Li et al., 2012 ANK3 as a risk gene for schizophrenia: new data in Han Chinese and meta analysis. American journal of medical genetics. Part B, Neuropsychiatric genetics : the official publication of the International Society of Psychiatric Genetics 159B: 997-1005.

Zhao, X., D. Lenek, U. Dag, B. J. Dickson and K. Keleman, 2018 Persistent activity in a recurrent circuit underlies courtship memory in Drosophila. Elife 7.

Zhao, X., T. Sternsdorf, T. A. Bolger, R. M. Evans and T. P. Yao, 2005 Regulation of MEF2 by histone deacetylase 4- and SIRT1 deacetylase-mediated lysine modifications. Molecular and cellular biology 25: 8456-8464.

Table 1. Frequency of mushroom body phenotypes resulting from knock down of Ank2. The percentage of brains displaying each phenotype was calculated from the total number of brains analyzed for each genotype $(n)$ at 22,25 and $30^{\circ} \mathrm{C}$. Statistical analysis was performed with Fisher's Exact Test. Knockdown of Ank2 resulted in significantly more brains with thin lobes $(p=0.0032)$, elongation and guidance deficits $(p=0.0032)$, or absent $\alpha$ and/or $\beta$ lobes $(p=0.0014)$, than controls at $25^{\circ} \mathrm{C}$. The more severe phenotypes of completely absent $\alpha$ and/or $\beta$ lobes were more pronounced at higher temperatures $\left(27^{\circ} \mathrm{C}\right.$ compared to $\left.22^{\circ} \mathrm{C}, p=0.0015\right)$.

\begin{tabular}{|c|c|c|c|c|}
\hline Genotype & elav $>w(C S 10)$ & elav> Ank2 RNAi & elav> Ank2 RNAi & elav> Ank2 RNAi \\
\hline Temperature & $25^{\circ} \mathrm{C}$ & $22^{\circ} \mathrm{C}$ & $25^{\circ} \mathrm{C}$ & $27^{\circ} \mathrm{C}$ \\
\hline$n$ & 20 & 30 & 30 & 16 \\
\hline Fused $\beta$ lobes & $0 \%$ & $13 \%$ & $10 \%$ & $0 \%$ \\
\hline
\end{tabular}


Figure 1 made available under aCC-BY 4.0 International license.

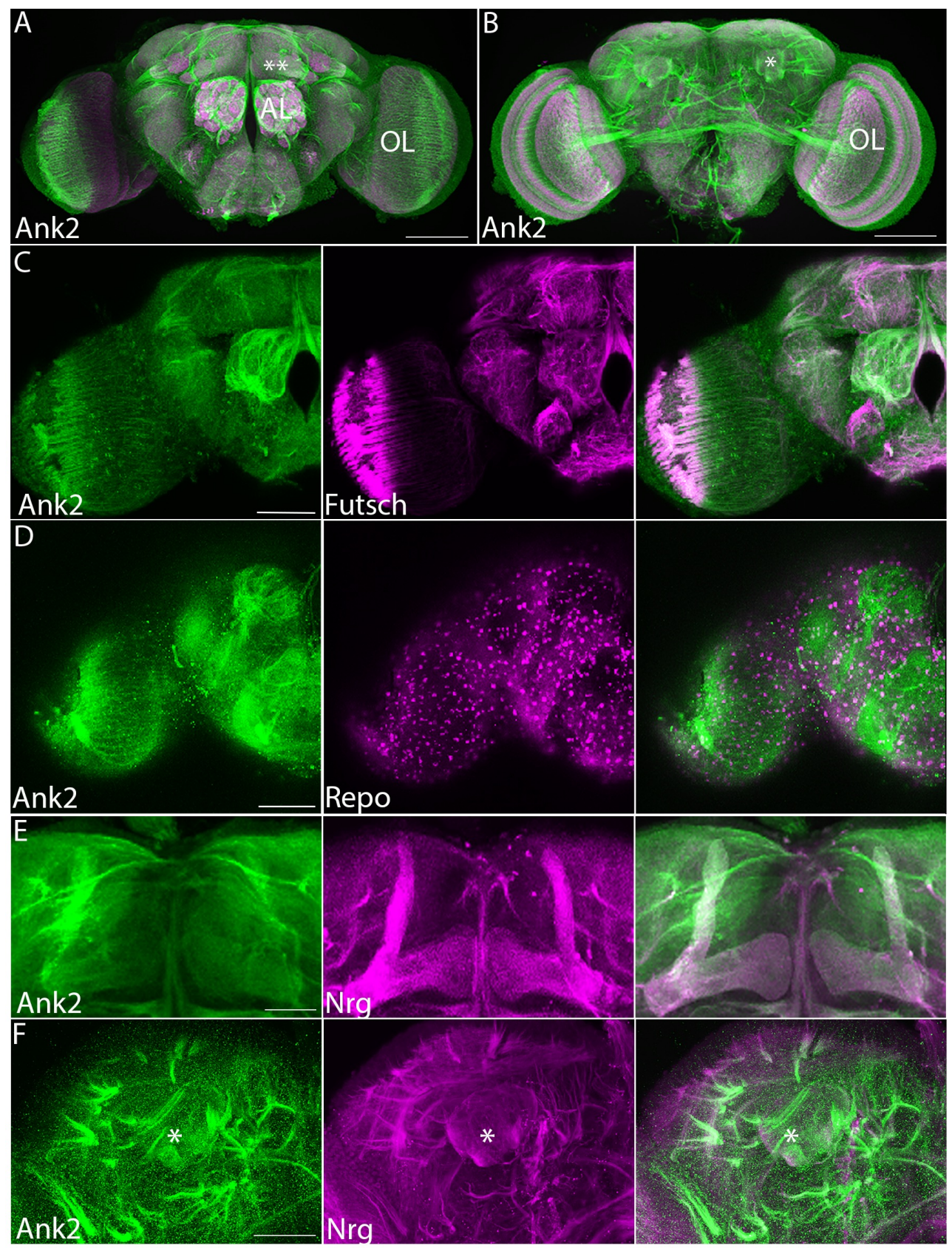

Figure 1. Expression of Ank2 in the adult brain. Immunohistochemistry on whole mount wild-type brains indicates widespread distribution of Ank2-L. A,B Confocal projection of brains labelled with Ank2-L (green) and nc82 (Bruchpilot, magenta) antibodies, to highlight the synaptic neuropil. Images are Z-stacks of $1 \mu \mathrm{m}$ optical sections. A, Anterior confocal projection. AL, antennal lobe, $\mathrm{OL}$ optic lobe, $* *$ indicates the $\gamma$ lobe of the mushroom body. Scale bar $=100 \mu \mathrm{m}$ B-F. Co-labelled proteins are shown in magenta and labelled in the middle panel. B. Posterior confocal projection, *indicates the calyx of the mushroom body. Scale bar $=100$ $\mu \mathrm{m}$. C. Immunohistochemistry with Ank2-L and 22C10 (Futsch) antibodies showing codistribution in neurons, with widespread localization to axon tracts. Scale bar $=50 \mu \mathrm{m}$. D. Ank2-L does not codistribute with pan-glial marker Repo. Scale bar $=50 \mu \mathrm{m}$. E. Ank2-L colocalizes with $\mathrm{Nrg}$ in the mushroom body lobes. Scale bar $=50 \mu \mathrm{m}$. F. Ank2-L also codistributes with $\mathrm{Nrg}$ in axon tracts surrounding the calyx (asterisk). Scale bar $=50 \mu \mathrm{m}$. 
bioRxiv preprint doi: https://doi.org/10.1101/2021.07.18.452850; this version posted July 18, 2021. The copyright holder for this preprint

(which was not certified by peer review) is the author/funder, who has granted bioRxiv a license to display the preprint in perpetuity. It is Figure 2 made available under aCC-BY 4.0 International license.
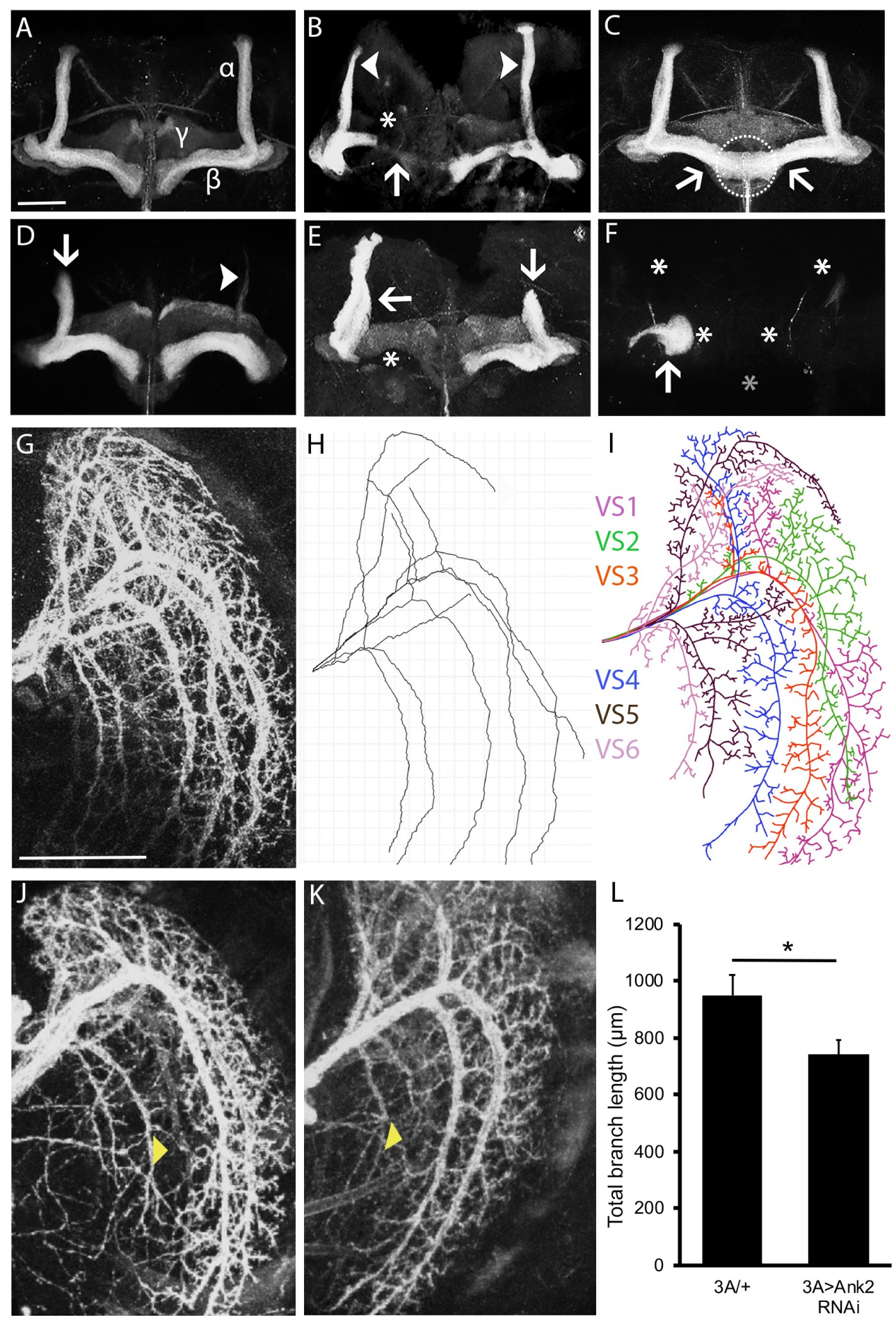
Figure 2. Reduced Ank2 expression disrupts neuronal development. A-F. Immunohistochemistry with anti-Fasll on whole mount brains reveals morphological defects of the mushroom body resulting from pan-neuronal expression of Ank2 RNAi driven by elav-GAL4. Knockdown was confirmed by RT-qPCR (as described in the methods). All images are frontal confocal projections through the mushroom body. Scale bar $=50 \mu \mathrm{m}$. A. Wild-type mushroom body stained with anti-Fas II to highlight the $\alpha, \beta$ and $\gamma$ lobes. B. Thin $\alpha$ lobes (arrowheads), a prematurely terminated $\beta$ lobe (arrow) and a missing $\gamma$ lobe (asterisk). C. $\beta$ lobes (arrows) have crossed the midline and appear fused (circle). D. A thin (arrowhead) and a prematurely terminated $\alpha$ lobe (arrow). E. Misoriented $\beta$ lobe (arrow) which projects anteriorly rather than its usual medial orientation (asterisk). A prematurely terminated $\alpha$ lobe is also present (arrow). F. None of the lobes have elongated (asterisks) and axon stalling is observed whereby the axons grow in a ball-like structure (arrow). Grey asterisk indicates the midline. G-J. Immunohistochemistry on whole mount brains with anti-GFP driven by $3 A-G A L 4$ to detect Lifeact in LPTCs whole mount brains. All images are confocal projections through the optic lobe of the brain. G. The dendritic arbor of the six neurons comprising the vertical system of LPTCs in a wild-type brain is visualised with anti-GFP. Scale bar $=50 \mu \mathrm{m}$. H. Dendritic trace generated using SNT Tracer (Image J). I. Cartoon trace of the confocal micrograph showing the dendritic branching of each of the vertical system neurons. J,K. Knockdown of Ank2 results in defects in dendritic branching (arrow heads). L. Total dendrite branch length was reduced by knockdown of Ank2 (student's $t$-test $\mathrm{t}_{(36)}=2.27, \mathrm{p}<0.05$ ). 
bioRxiv preprint doi: https://doi.org/10.1101/2021.07.18.452850; this version posted July $18,2021$. The copyright holder for this preprint

(which was not certified by peer review) is the author/funder, who has granted bioRxiv a license to display the preprint in perpetuity. It is made available under aCC-BY 4.0 International license.

Figure 3
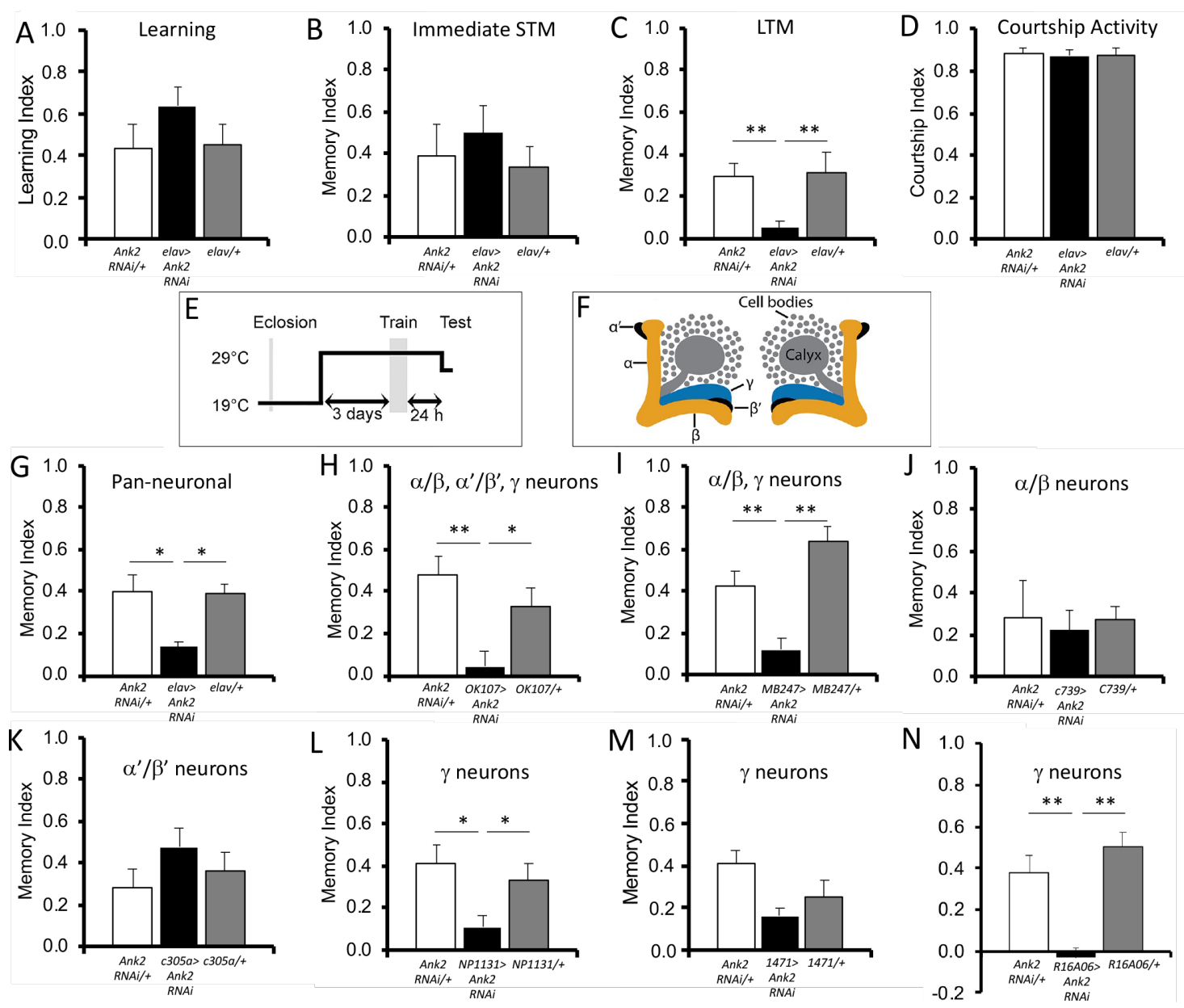
Figure 3. Ank2 is required for long-term memory. Learning and memory were assessed with the courtship suppression assay. The controls included in each assay are the GAL4 driver (plus tubP-GAL80ts where indicated) crossed to CS, and UASAnk2 crossed to $C S$, such that the progeny are heterozygous for either the driver or the RNAi. A-D. elav-GAL4 and UAS-Ank2 RNAi flies were crossed to achieve panneuronal knockdown of $A n k 2$ in progeny. A. Learning was unaffected knockdown of Ank2 (ANOVA, $\mathrm{F}_{(2,47)}=0.002, \mathrm{p}=0.252$ ). B. Immediate memory was also unaffected (ANOVA, $\left.\mathrm{F}_{(2,45)}=0.044, \mathrm{p}=0.819\right)$. C. Ank2 knockdown impaired long-term memory (ANOVA, $\mathrm{F}_{(2,60)}=7.31, \mathrm{p}<0.001$; post-hoc Tukey's HSD, $\left.* * \mathrm{p}<0.01\right)$. D. Courtship activity was not impaired by pan-neuronal knockdown of Ank2 (ANOVA, $\mathrm{F}_{(2,51)}=0.14$, $\mathrm{p}=0.870$ ). E-K. Knockdown of Ank2 in the adult mushroom body impairs LTM. Ank2 was knocked down in specific regions of the brain by crossing Ank2 RNAi to the indicated driver line and tubP-Gal80ts. E. Schematic diagram depicting the induction of expression in the adult mushroom body. Expression was restricted to the adult brain by raising flies at raised at $19^{\circ} \mathrm{C}$, at which temperature GAL80 represses GAL4. After eclosion, when flies were $3-5$ days old, the temperature was raised to $30^{\circ} \mathrm{C}$ for 72 hours, after which training commenced. At this temperature GAL80 is inactivated, allowing GAL4 to induce transgene expression. Twenty-four hours after training, the flies were equilibrated to $25^{\circ} \mathrm{C}$ for one hour prior to testing. F. Schematic diagram labelling the lobes of the mushroom body in which Ank2 was knocked down. G. Pan-neuronal knockdown of Ank2 in the adult brain impairs long term memory (ANOVA, $\mathrm{F}_{(2,54)}=0.317, \mathrm{p}<0.01$; post-hoc Tukey's HSD, ${ }^{*} \mathrm{p}<0.05$ ). $\mathrm{H}$. Similarly, memory is also impaired when knockdown of Ank2 is restricted to the mushroom body (ANOVA, $\mathrm{F}_{(2,52)}=0.922$, $\mathrm{p}<0.001$; post-hoc Tukey's HSD, ${ }^{*} \mathrm{p}<0.01$, $\left.{ }^{*} p<0.05\right)$. I. When knockdown is restricted to the $\alpha / \beta$ and $\gamma$ neurons of the mushroom body, long-term memory is still disrupted (ANOVA, $F_{(2,51)}=0.923$, $p<0.0001$; post-hoc Tukey's HSD, $\left.{ }^{* *} p<0.01\right)$. J. Reduction of $A n k 2$ in just the $\alpha / \beta$ neurons has no significant effect on long-term memory (ANOVA, $F_{(2,41)}=0.025$, $\mathrm{p}=0.819)$. K. There is also no impairment when Ank2 is reduced in the $\alpha^{\prime} / \beta^{\prime}$ neurons (ANOVA, $\mathrm{F}_{(2,51)}=0.122 \mathrm{p}=0.372$ ). L. Ank2 is required in the $\gamma$ lobes, as knockdown with NP1131-GAL4 impairs LTM (ANOVA, $\mathrm{F}_{(2,46)}=0.312$, $\mathrm{p}<0.01$; post-hoc Tukey's HSD, $\left.{ }^{*} \mathrm{p}<0.05\right)$. M. The weaker $\gamma$ lobe driver 1471-GAL4 reduced LTM, however this was not quite significant (ANOVA, $F_{(2,59)}=0.210, p=0.056$ ). Knockdown of Ank2 with the stronger $\gamma$ lobe driver R16A06-GAL4 did impair LTM significantly (ANOVA, $\mathrm{F}_{(2,33)}=18.57, \mathrm{p}<0.0001$, post-hoc Tukey's HSD, $\left.* * p<0.01\right)$. 

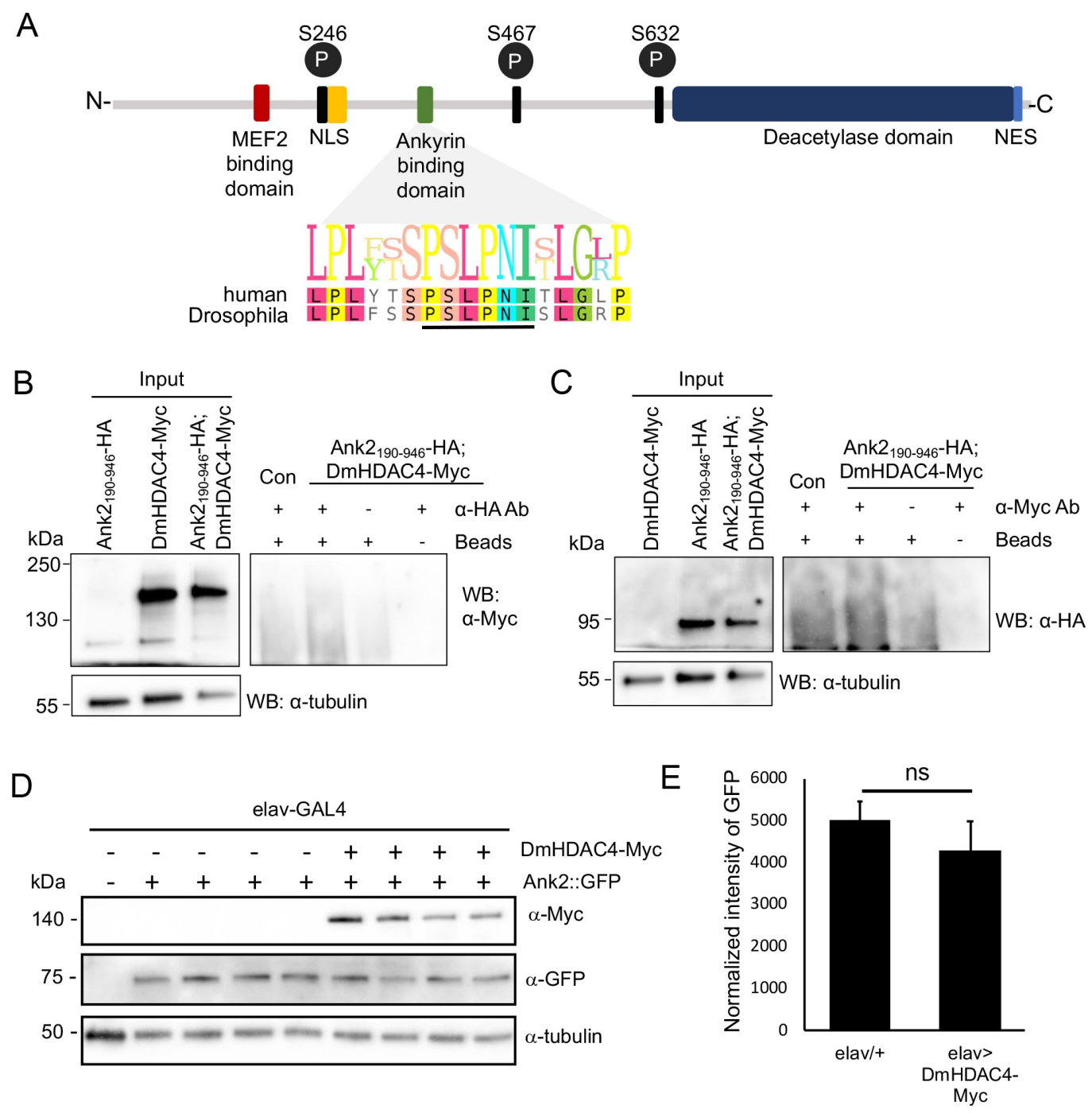

Figure 4. Ank2 does not bind HDAC4 and nor is its expression regulated by HDAC4. A. Domain structure of human HDAC4 showing binding sites conserved between Drosophila and human HDAC4. The amino acid sequence of the region containing the RFXANK/ANKRA2 binding site in human HDAC4 is shown, with the corresponding amino acid sequence in Drosophila HDAC4. NLS, nuclear localisation sequence, NES, nuclear export sequence. Ps circled in black are serine residues that when phosphorylated provide binding sites for 14-3-3 mediated nuclear export. B,C. Co-immunoprecipitation of Ank2190-946-HA and DmHDAC4Myc from whole cell lysates of fly heads with either anti-Myc or anti-HA. Both blots were probed with anti-tubulin as a loading control. Input samples $=30 \mu \mathrm{g}$. B. Following immunoprecipitation with anti-HA, DmHDAC4-Myc was not detected upon probing with anti-Myc. Inputs include Ank2190-946-HA, which is not detected by Myc, DmHDAC4-Myc, and Ank2190-946-HA; DmHDAC4-Myc to confirm expression. C. In the reciprocal experiment, flies expressing Ank2190-946HA; DmHDAC4-Myc were subjected to IP with anti-Myc, however Ank2190-946HA was not detected upon probing with anti-HA. D. elav-GAL4; Ank2::GFP flies were crossed to $w(C S) 10$ and UAS-DmHDAC4-Myc and whole head lysates of progeny were generated for western blotting. Samples were processed from four independent crosses. Blots were probed with anti-Myc to verify expression of DmHDAC4-Myc and anti-GFP to determine whether the amount of Ank2::GFP normalized to tubulin is altered in the presence of DmHDAC4. E. There was no significant change in the level of Ank2::GFP on expression of DmHDAC4-Myc. 
bioRxiv preprint doi: https://doi.org/10.1101/2021.07.18.452850; this version posted July 18, 2021. The copyright holder for this preprint (which was not certified by peer review) is the author/funder, who has granted bioRxiv a license to display the preprint in perpetuity. It is made available under aCC-BY 4.0 International license.

\section{Figure 5}
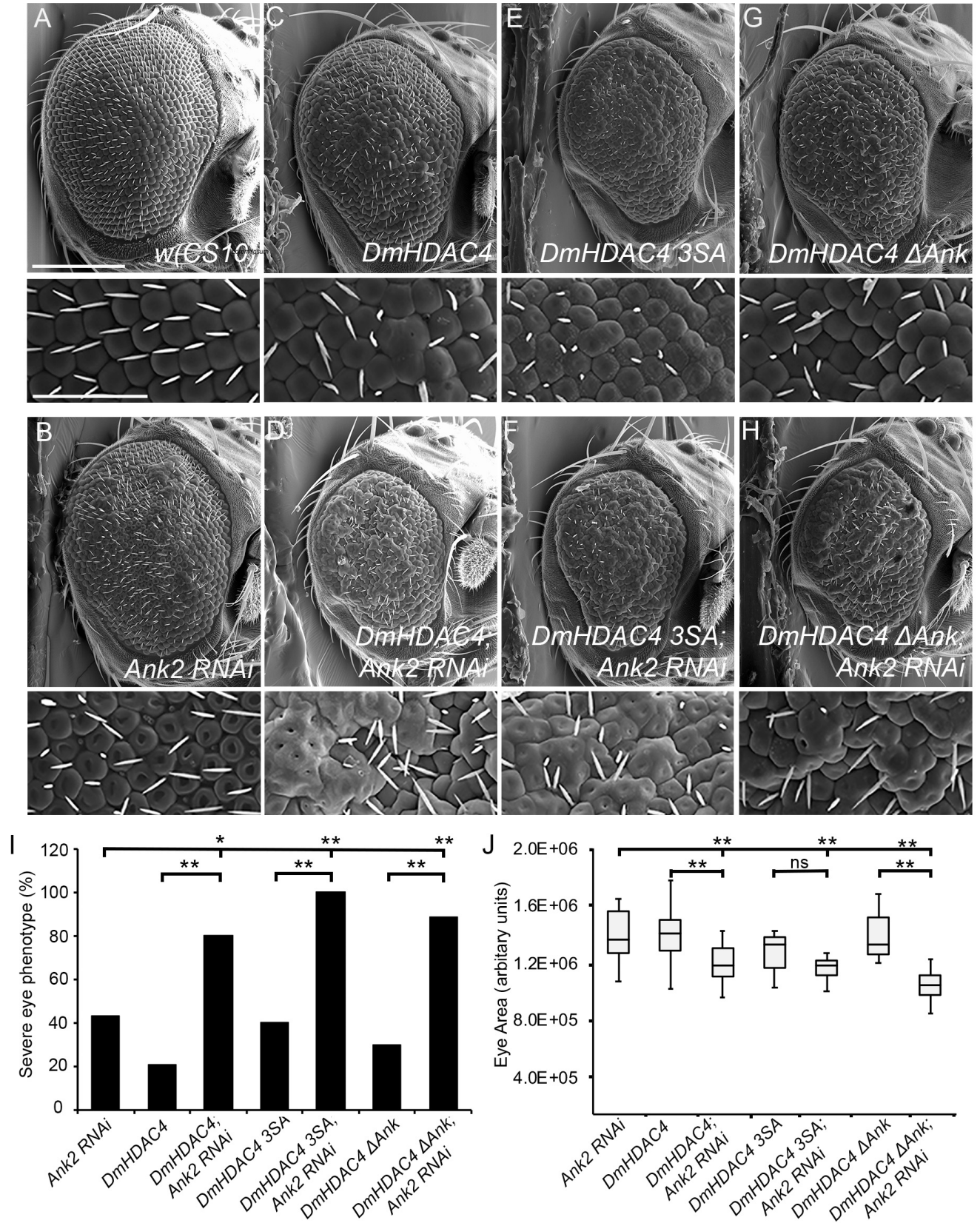
Figure 5. Ank2 interacts genetically with HDAC4 in the eye. A-H. Scanning electron micrographs of Drosophila eyes expressing Ank2 RNAi and/or HDAC4 variants. The genotypes indicated in each panel were generated by crossing GMR-GAL4 females to males carrying each UAS-HDAC4 construct or Ank2 RNAi to the w(CS10) control. Top panel: Scale bar $=200 \mu \mathrm{m}$. Bottom panel: Scale bar $=100 \mu \mathrm{m}$. I. The percentage of eyes displaying severe phenotypes are shown for each genotype. Severe phenotypes were scored as severe disorganisation with fused ommatidia in more than ten areas, and/or more than 50 collapsed ommatidia with crevices or cavities. ${ }^{*} \mathrm{p}<0.05, * * \mathrm{p}<0.01$ following one-tailed Fisher' $\mathrm{s}$ exact test. $\mathrm{p}$-values: Ank2 RNAi:DmHDAC4; Ank2 RNAi $=0.0247$, Ank2 RNAi:DmHDAC4 3SA; Ank2 RNAi = 0.0006, Ank2 RNAi:DmHDAC4 $\triangle A n k ;$ Ank2 RNAi = 0.0028, DmHDAC4:DmHDAC4; Ank2 RNAi $=0.0004$, DmHDAC4 3SA:DmHDAC4 3SA; Ank2 RNAi $=0.0005$, DmHDAC4 $\triangle A n k: D m H D A C 4 \triangle A n k 2 ; A n k 2 \mathrm{RNAi}=0.0002$. J. Eye sizes were quantified by tracing a line around each eye and calculating the area in arbitrary units using ImageJ software and plotting on Box and whisker plots to show the variation in eye size for each genotype. ${ }^{* *} p<0.01$ following one-way ANOVA and post-hoc Tukey's HSD test for significance. $\mathrm{p}$-values: $w(\operatorname{cs} 10):$ Ank2 RNAi $=0.007, w(\operatorname{cs} 10): D m H D A C 4=0.009$, $w(c s 10): D m H D A C 4 ;$ Ank2 RNAi $=0.001$, Ank2 RNAi:DmHDAC4; Ank2 RNAi $=0.001$, DmHDAC4:DmHDAC4; Ank2 = 0.001, $w(\operatorname{cs10):DmHDAC4} 3 A=0.001$, $w(\operatorname{cs} 10):$ DmHDAC4 3A; Ank2 RNAi $=0.001$, DmHDAC4 3A:DmHDAC4 3A; Ank2 RNAi = 0.285, w(cs10):DmHDAC4 $\triangle A n k=0.001, w(c s 10): D m H D A C 4 \triangle A n k ;$ Ank2 RNAi $=$ 0.001, DmHDAC4 $\triangle A n k: D m H D A C 4 \triangle A n k ; A n k 2 \mathrm{RNAi}=0.001 . \mathrm{n}=$ number of eyes per sample. The number of eyes analyzed per genotype is indicated in Table S1. 